Transfus Med Hemother 2011;38:396

DOI: $10.1159 / 000334866$
Received: October 24, 2011

Accepted: October 26, 2011

Published online: November 14, 2011

\title{
Erwiderung zum Kommentar von Dr. Sascha Rolf Lüder: Transfus Med Hemother 2011;38(6): DOI: 10.1159/000334844
}

\author{
Friedger von Auer \\ Königswinter, Deutschland
}

1. Die in Deutschland seit Langem geübte Praxis der Zahlung einer pauschalierten Aufwandsentschädigung bei der Blutspende entspricht vollkommen dem Sinn und Zweck des $§ 10$ Transfusionsgesetz (TFG) in Verbindung mit dem nach wie vor gültigen Votum 1 des Arbeitskreises Blut (AK Blut) vom 15. November 1993. Nach $\S 10$ Satz 2 TFG kann der spendenden Person eine Aufwandsentschädigung gewährt werden, die sich an dem unmittelbaren Aufwand je nach Spendeart orientieren soll. Diese an dem genannten AK-Blut-Votum orientierte Regelung sollte die schon damals von den Blutspendediensten praktizierte Zahlung einer pauschalierten Aufwandsentschädigung nicht in Frage stellen. In der Begründung zu der heute gültigen Fassung von $\S 10$ TFG wird auf das Votum 1 des AK Blut ausdrücklich Bezug genommen, das für die Aufwandsentschädigung zur Blutspende einen Höchstsatz vorgibt, damit der Charakter als Aufwandsentschädigung gewahrt bleibt. Diese Vorgabe kann nicht als überzogen angesehen werden und wird von den Blutspendediensten eingehalten. Die zuständigen Behörden überwachen die Praxis der Gewährung einer Aufwandsentschädigung.

2. Was unter dem Begriff «unmittelbarer Aufwand» in $\S 10$ Satz 2 TFG zu verstehen ist, ergibt sich aus der Begründung zu der Regelung. Dort werden in Anlehnung an das genannte AKBlut-Votum jedenfalls die «Fahrtkosten und/oder der Zeitaufwand» genannt. Zeitaufwand ist jedenfalls die Dauer der Spende, die bei der Vollblutspende geringer ist als bei anderen Spendearten. Deshalb ist auch nur eine geringe Aufwandsentschädigung vorgesehen. Das Wort «jedenfalls» in der Begründung deutet an, dass auch noch ein anderer Aufwand berücksichtigt werden kann. Hier kommt ein immaterieller Aufwand in Betracht, der darin besteht, dass sich die spendende Person zu einem Eingriff in ihren Körper zur Spendeentnahme bereit erklärt. Die Aufwandsentschädigung hat dann auch den Charakter einer Anerkennung für den Dienst der spendenden Person am Nächsten, wie sie bei vielen Blutspendediensten auch durch die Gabe kleiner Geschenke zum Ausdruck kommt. Der Gedanke der Anerkennung ist in der Empfehlung des Europa- rates R (95) 14 für die unentgeltliche Spende ausdrücklich enthalten. In der Begründung zu $§ 10$ Satz 2 TFG wird eine «Pauschalierung der Aufwandsentschädigung» für zulässig gehalten.

3. Die Blutrichtlinie 2002/98/EG der Europäischen Union (EU) ist die allein relevante Rechtsgrundlage für das innerstaatliche Recht der EU-Mitgliedstaaten. Sie enthält in ihrem verfügenden Teil keine zwingende Regelung zur Unentgeltlichkeit oder Bezahlung der Blutspende. Dazu hat die EU nach dem einschlägigen EG-Vertrag (heute: Vertrag über die Arbeitsweise der Europäischen Union - AEUV) keine Regelungskompetenz. Das in dem Erwägungsgrund 23 zu der Richtlinie angeführte Argument eines hohen Qualitäts- und Sicherheitsstandards für Blut und Blutbestandteile durch die unbezahlte Blutspende mag möglicherweise für bezahlte Spenden zutreffen. Für die aufwandsentschädigte Blutspende im Sinne der deutschen Praxis trifft dieses Argument nicht zu. Das wird in Votum 26 des AK Blut aus dem Jahr 2001 ausdrücklich festgestellt und in dem Urteil des EuGH bestätigt. Dass auch die Empfehlung des Europarates nicht zu der deutschen Regelung und Praxis der Aufwandsentschädigung in Widerspruch steht, ist bereits ausgeführt worden. Im Übrigen könnten die Erstattung von Fahrtkosten und die Gewährung von Anerkennungen oder kleinen Geschenken, was die Empfehlung des Europarates ausdrücklich zulässt, zusammengenommen einen höheren Wert darstellen, als er in Deutschland tatsächlich zulässig ist.

4. Soweit ersichtlich, wird die Praxis der Zahlung einer pauschalierten Aufwandsentschädigung in Deutschland nur im Zusammenhang mit der Vollblutspende in Frage gestellt. Im Hinblick auf die Plasma- und die Trombozythapherese zahlen alle Spendedienste pauschalierte Geldbeträge als Aufwandsentschädigung, die teilweise höher sind als die Beträge für die Vollblutspende. Das lässt das TFG zu, wie die differenzierte Regelung des $\S 10$ Satz 2 TFG zeigt. Was in diesen Fällen nach geltendem Recht als zulässig und auch praktikabel angesehen wird, gilt für die Vollblutspende gleichermaßen. Eine diesbezügliche Differenzierung hat der Gesetzgeber nicht vorgesehen und wäre rechtlich auch kaum zu begründen.

\section{KARGER}

Fax +497614520714

Information@Karger.de

www.karger.com (c) 2011 S. Karger GmbH, Freiburg

Accessible online at:

www.karger.com/tmh
Friedger von Auer

Kelvinstraße 12

53639 Königswinter, Deutschland

FvAuer@aol.com 